\title{
Clinical Features and Long-term Prognosis of Retinoblastoma according to Age at Diagnosis
}

\author{
Hyun-Ah Kim, Young Hee Yoon, June-Gone Kim, Joo Yong Lee \\ Department of Ophthalmology, Asan Medical Center, University of Ulsan College of Medicine, Seoul, Korea
}

\begin{abstract}
Purpose: We aimed to study the clinical characteristics and long-term prognoses of retinoblastoma according to the age at diagnosis.

Methods: A retrospective chart review of non-screened patients newly diagnosed with retinoblastoma between January 2007 and February 2018.

Results: Among the 20 patients analyzed, 11 were diagnosed at an age younger than 1 year (group 1) and nine at 1 year or older (group 2). The mean lag times until diagnosis were $1.0 \pm 0.4$ and $5.0 \pm 2.1$ months for groups 1 and 2 , respectively $(p=0.056)$. The mean follow-up durations were $49.4 \pm 12.7$ and $58.3 \pm 8.8$ months, respectively $(p=0.412)$. Group 1 had a significantly higher proportion of bilateral retinoblastoma than did group 2 (72.7\% vs. $11.1 \%, p=0.010)$. Four of five patients (80.0\%) with germline $R B 1$ mutations were diagnosed with retinoblastoma at age 3 months or younger. The eyes of patients in group 2 had significantly higher International Intraocular Retinoblastoma Classification stages than did those of patients in group 1 ( $p$ for trend $=0.010$ ). The proportion of eyes with optic nerve invasion and those that had undergone enucleation were significantly higher in group 2 ( $p=0.033$ and 0.046 , respectively). Survival did not differ according to the age at diagnosis.

Conclusions: Early onset retinoblastoma does not seem to indicate poor ocular or survival prognosis in Korean children with retinoblastoma.
\end{abstract}

Key Words: Child, Diagnosis, Neoplasms, Prognosis, Retinoblastoma

Retinoblastoma is the most common primary intraocular malignancy in childhood. The incidence of retinoblastoma has been reported to be 11.2 per 100,000 children aged 0 to 4 years in Korea [1], which is similar to the incidences in the USA and Europe [2,3]. Owing to easy accessibility and well-performed healthcare for infants and children, the

Received: August 6, 2019 Final revision: February 24, 2020

Accepted: March 11, 2020

Corresponding Author: Joo Yong Lee, MD, PhD. Department of Ophthalmology, Asan Medical Center, University of Ulsan College of Medicine, 88 Olympic-ro 43-gil, Songpa-gu, Seoul 05505, Korea. Tel: 82-23010-3976, Fax: 82-2-470-6440, E-mail: ophthalmo@amc.seoul.kr

This study was presented as a poster presentation at the Asia-Pacific Vitreo-retina Society, December 14-16, 2018, Seoul, Korea. mortality rate of patients diagnosed with retinoblastoma in Korea has decreased to $4.5 \%$ during 2001 to 2010 [1], which is not much different from the mortality rate of $3 \%$ to $5 \%$ reported in other developed countries [3].

The development of retinoblastoma is initiated by the biallelic inactivation of the tumor suppressor $R B 1$ gene located on chromosome 13q14. Studies have shown that $10 \%$ of patients with retinoblastoma inherit a family-specific RB1 mutation from a parent, while the other $90 \%$ develop retinoblastoma sporadically [4]. Among these patients, $30 \%$ have a sporadic germline mutation, and the remaining $60 \%$ have non-heritable retinoblastoma with two somatic mutations [4]. For children with a family history of retinoblastoma, early screening is usually done before the symp- 
toms appear, because early diagnosis results in a better prognosis $[5,6]$. However, those with spontaneous germline or somatic mutations are likely to be detected at a later stage, as they are usually diagnosed only after developing symptoms [7].

The majority of patients with germline mutations present with multifocal bilateral retinoblastoma, usually before 1 year of age, while those with two somatic mutations always have unilateral retinoblastoma and are diagnosed around 2 years of age [8]. Other cancers caused by germline mutations, such as the BRCA1/2 mutation in breast cancer, are often related to a worse prognosis $[9,10]$. A few prior studies have examined the survival rates of retinoblastoma patients according to the patient's age at diagnosis. A study using the Korean National Cancer Registry Database reported no difference in survival between patients diagnosed at age 1 year or younger and at age 2 years or older [1]. Likewise, a study in Mexico showed no difference in survival rates between patients diagnosed at birth to 6 months and at 6 to 12 months [7].

Although survival is the most important factor to consider, since the survival rates of patients with retinoblastoma in Korea and other developed countries are relatively high, other factors such as disease stage and the possibility of eye preservation also need attention. Despite their possible clinical significance, these factors have not been addressed according to the age at diagnosis in previous studies. Therefore, this study aimed to examine the clinical characteristics and long-term prognosis of retinoblastoma, focusing on the possible differences according to the age at diagnosis.

\section{Materials and Methods}

\section{Patient samples}

This study was a retrospective chart review of patients initially diagnosed with retinoblastoma at a single institute in Korea between January 2007 and February 2018. Only patients who were followed up for 6 months or more were included for analysis.

\section{Diagnosis and staging of retinoblastoma}

The diagnosis of retinoblastoma was based on clinical examination findings or pathological examination results.
All patients had documented color fundus photographs acquired using RetCam II (Clarity Medical Systems, Pleasanton, CA, USA) or a fundus camera (AFC-330, Nidek, Gamagori, Japan; or TRC-NW6S, Topcon Corporation, Tokyo, Japan). Fluorescein angiography and B-scan ultrasonography were performed for differential diagnosis and to determine the tumor extent if clinically required. Computerized tomography and magnetic resonance imaging of the orbit were performed to evaluate tumor location and size, the presence of calcifications, and optic nerve and extraocular involvement. A bone scan was performed to evaluate the presence of bone metastasis. The International Intraocular Retinoblastoma Classification (IIRC) was used for staging [11].

\section{Genetic analysis}

All patients initially diagnosed with retinoblastoma under the age of 5 years were recommended to undergo genetic evaluation to identify germline $R B 1$ mutations. In our institution, only fluorescence in situ hybridization (FISH), which detects large deletions, and multiplex ligation-dependent probe amplification (MLPA), which detects large deletions and duplications, were available. Since our laboratory did not conduct $R B 1$ gene sequence analysis, which detects small deletions, insertions, and point mutations, polymerase chain reaction (PCR)-direct sequencing was commissioned at another institute when needed. After obtaining informed consent from the patients, FISH (Cytocell, Cambridge, UK; or Vysis, Abbott Park, IL, USA), MLPA (MRC Holland, Amsterdam, the Netherlands), or PCR-direct sequencing were performed alone or in combination according to the clinician's decision at the time of diagnosis.

\section{Data collection}

Data collected from the clinical notes included patients' family histories of retinoblastoma, sex, initial symptom or sign, age at onset of initial sign or symptom, age at diagnosis, duration of follow-up, laterality, best-corrected visual acuity (BCVA) at the initial and final visits, genetic evaluation results, IIRC group, radiological and pathological findings, and treatment modalities. The presence of optic nerve invasion, foveal involvement, and distant metastasis was evaluated on the basis of fundus, radiological, and pathological examination results. 


\section{Statistical analysis}

Statistical analyses were conducted using IBM SPSS Statistics ver. 21.0 (IBM Corp., Armonk, NY, USA) or SAS ver. 9.4 (SAS Inc., Cary, NC, USA). Lag time was calculated as the interval between the age at the onset of symptom or sign and the age at diagnosis. All data are presented as mean \pm standard error for continuous variables and number (\%) for categorical variables. The Mann-Whitney test was used to analyze continuous variables, and Fisher exact test was used to analyze categorical variables. The Cochran-Armitage test for trend was used to assess the trends across IIRC groups according to the age at diagnosis. Spearman's rank correlation analysis and logistic regression analysis were carried out to examine the associations between variables. A $p$-value less than 0.05 was considered statistically significant.

\section{Ethics statement}

The study protocol was reviewed and approved by the institutional review board of Asan Medical Center (20180383) and was conducted in adherence to the tenets of the
Declaration of Helsinki. For this type of study, informed consent was not required.

\section{Results}

In this study, out of 28 patients diagnosed with retinoblastoma during the study period, eight were excluded because of loss to follow-up within 6 months, and the remaining 20 patients were selected for analysis. Of them, 11 were diagnosed with retinoblastoma at an age younger than 1 year (group 1) and nine at age 1 year or older (group 2). None of the patients had a family history of retinoblastoma, and therefore none were detected by screening. The demographic and clinical characteristics according to patient age at diagnosis are shown in Table 1. The mean and median ages at onset of sign or symptom were significantly younger in group 1 compared with group 2: $3.0 \pm 0.7$ vs. $29.2 \pm 9.2$ months; 3 months (interquartile range [IQR], $1-5$ months) vs. 17 months (IQR, 14-27 months), respectively, with $p<0.001$ for both comparisons. Similarly, the mean and median ages at diagnosis were also significantly younger in group 1 than in group $2(4.0 \pm 0.9$ vs. $34.3 \pm$

Table 1. Demographic and clinical characteristics of retinoblastoma according to age at diagnosis

\begin{tabular}{lccc}
\hline Characteristics & Group $1^{*}(\mathrm{n}=11)$ & Group 2 $(\mathrm{n}=9)$ & $p$-value \\
\hline Sex & & & 0.092 \\
Male & $7(63.6)$ & $2(22.2)$ & \\
Female & $4(36.4)$ & $7(77.8)$ & \\
Age at onset of sign or symptom (mon) & $3.0 \pm 0.7$ & $29.2 \pm 9.2$ & $<0.001$ \\
Age at diagnosis (mon) & $4.0 \pm 0.9$ & $34.3 \pm 10.0$ & $<0.001$ \\
Lag time (mon) & $1.0 \pm 0.4$ & $5.0 \pm 2.1$ & 0.056 \\
Duration of follow-up (mon) & $49.4 \pm 12.7$ & $58.3 \pm 8.8$ & 0.412 \\
Initial manifesting sign or symptom & & & 0.056 \\
Leukocoria & $3(27.3)$ & $6(66.7)$ & \\
Strabismus & $4(36.4)$ & $1(11.1)$ & \\
Orbital swelling & $1(9.1)$ & $0(0)$ & 0.010 \\
Dysmorphic feature & $3(27.3)$ & $0(0)$ & \\
Decreased visual acuity & $0(0.0)$ & $2(22.2)$ & \\
Laterality & & $8(88.9)$ & \\
Unilateral & $3(27.3)$ & $1(11.1)$ & \\
$\quad$ Bilateral & $8(72.7)$ & & \\
\hline
\end{tabular}

Values are presented as number (\%) or mean \pm standard error.

${ }^{*}$ Age at diagnosis $<1$ year; ${ }^{\dagger}$ Age at diagnosis $\geq 1$ year. 
Table 2. Germline $R B 1$ mutation status, laterality, and initial manifesting sign or symptom according to the age at diagnosis

\begin{tabular}{|c|c|c|c|c|c|}
\hline Patient no. & $\begin{array}{l}\text { Age at diagnosis } \\
\text { (mon) }\end{array}$ & $\begin{array}{l}\text { Initial manifesting sign or } \\
\text { symptom }\end{array}$ & $\begin{array}{c}\text { Germline } R B 1 \\
\text { mutation }\end{array}$ & Method of genetic analysis & Laterality \\
\hline 1 & 0 & Dysmorphic features & Yes & FISH for $13 q 14$ & Bilateral \\
\hline 2 & 0 & Dysmorphic features & Yes & FISH for $13 \mathrm{q} 14$ & Bilateral \\
\hline 3 & 1 & Dysmorphic features & Yes & FISH for $13 \mathrm{q} 14$ & Bilateral \\
\hline 4 & 3 & Leukocoria & Nil & FISH for $13 \mathrm{q} 14$ & Bilateral \\
\hline 5 & 3 & Leukocoria & Yes & PCR-direct sequencing of $R B 1$ & Bilateral \\
\hline 6 & 4 & Leukocoria & - & - & Bilateral \\
\hline 7 & 5 & Strabismus & Nil & PCR-direct sequencing of $R B 1$ & Unilateral \\
\hline 8 & 7 & Strabismus & Nil & FISH for $13 q 14$ & Bilateral \\
\hline 9 & 7 & Strabismus & Nil & PCR-direct sequencing of $R B 1$ & Unilateral \\
\hline 10 & 7 & Strabismus & Nil & PCR-direct sequencing of $R B 1$ & Unilateral \\
\hline 11 & 8 & Orbital swelling & Nil & $\begin{array}{l}\text { PCR-direct sequencing of } R B 1 \\
+ \text { FISH for } 13 \mathrm{q} 14\end{array}$ & Bilateral \\
\hline 12 & 15 & Leukocoria & Yes & PCR-direct sequencing of $R B 1$ & Bilateral \\
\hline 13 & 15 & Leukocoria & Nil & $\begin{array}{l}\text { PCR-direct sequencing of } R B 1 \\
+ \text { FISH for } 13 \mathrm{q} 14\end{array}$ & Unilateral \\
\hline 14 & 16 & Leukocoria & Nil & PCR-direct sequencing of $R B 1$ & Unilateral \\
\hline 15 & 20 & Strabismus & Nil & PCR-direct sequencing of $R B 1$ & Unilateral \\
\hline 16 & 21 & Leukocoria & Nil & $\begin{array}{l}\text { PCR-direct sequencing of } R B 1 \\
+ \text { MLPA of } R B 1\end{array}$ & Unilateral \\
\hline 17 & 21 & Leukocoria & Nil & $\begin{array}{l}\text { PCR-direct sequencing of } R B 1 \\
+ \text { FISH for } 13 \mathrm{q} 14\end{array}$ & Unilateral \\
\hline 18 & 27 & Leukocoria & Nil & PCR-direct sequencing of $R B 1$ & Unilateral \\
\hline 19 & 85 & Decreased VA & - & - & Unilateral \\
\hline 20 & 89 & Decreased VA & - & - & Unilateral \\
\hline
\end{tabular}

$R B 1=$ retinoblastoma $1 ; \mathrm{FISH}=$ fluorescence in situ hybridization; $\mathrm{PCR}=$ polymerase chain reaction; $\mathrm{MLPA}=$ multiplex ligation-dependent probe amplification; $\mathrm{VA}=$ visual acuity.

10.0 months; 4 months [IQR, 1-7] vs. 21 months [IQR, 1627], respectively; $p<0.001$ for both comparisons). The mean lag times until diagnosis were $1.0 \pm 0.4$ and $5.0 \pm 2.1$ months, respectively, for groups 1 and $2(p=0.056)$. The mean durations of follow-up for groups 1 and 2 were 49.4 \pm 12.7 and $58.3 \pm 8.8$ months, respectively $(p=0.412)$. The most common initial manifesting sign or symptom was strabismus (36.4\%) in group 1 and leukocoria (66.7\%) in group 2. Sex, lag time until diagnosis, duration of follow-up, and type of initial sign or symptom did not differ according to the age at diagnosis. Group 1 had a significantly larger proportion of bilateral retinoblastoma $(72.7 \%$ vs. $11.1 \%, p=0.010$ ) than did group 2 .

Table 2 shows the germline $R B 1$ mutation status, laterality, and initial manifesting sign or symptom according to the age at diagnosis. Genetic evaluations were performed in 10 of $11(90.9 \%)$ patients in group 1 and 7 of $9(77.8 \%)$ patients in group 2. The parents of patient number 6 refused consent for the genetic test. Patient numbers 19 and 20 were diagnosed at age 85 and 89 months, respectively, and were not recommended for genetic analysis by the clinician. Out of the 17 patients who underwent genetic evaluation, five (29.4\%) were found to have a germline $R B 1$ mutation. Four of these five patients $(80.0 \%)$ with germline $R B 1$ mutations were diagnosed with retinoblastoma at age 3 months or younger. Genetic analyses for patient numbers 4,8 , and 11 could not detect germline $R B 1$ mutations, even though retinoblastoma was found in both eyes. Two of these three patients were only tested using FISH. Patient numbers 1,2 , and 3 with germline $R B 1$ mutations detected 
A
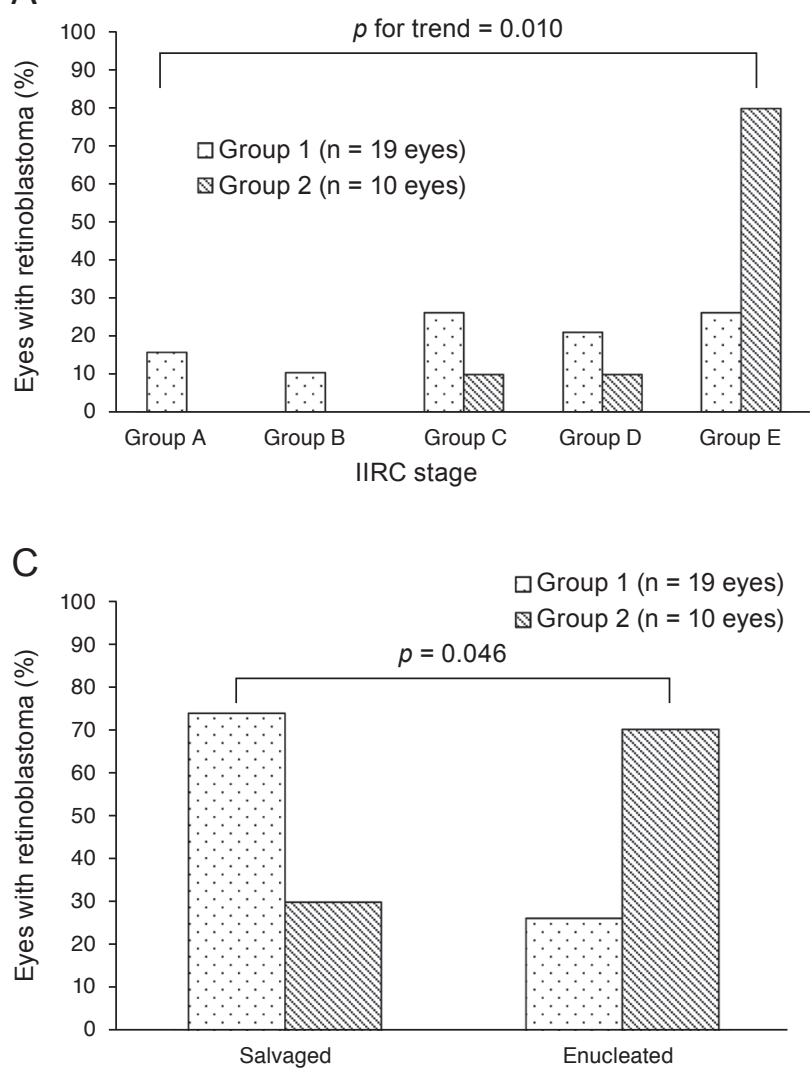

B

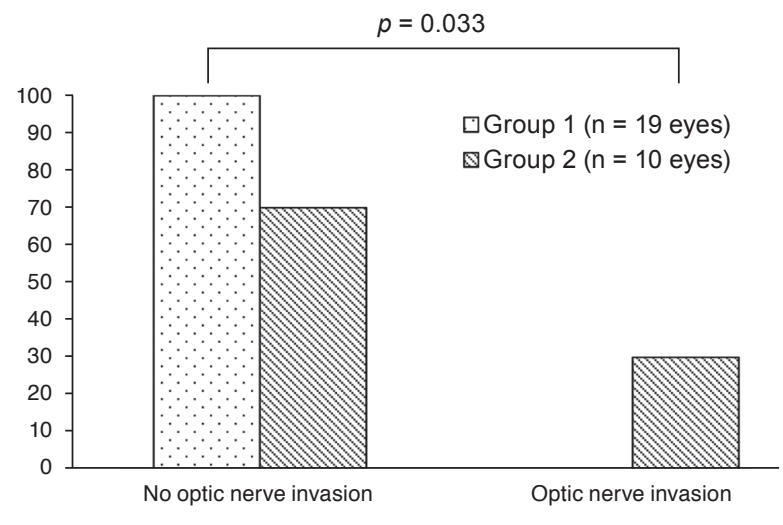

D

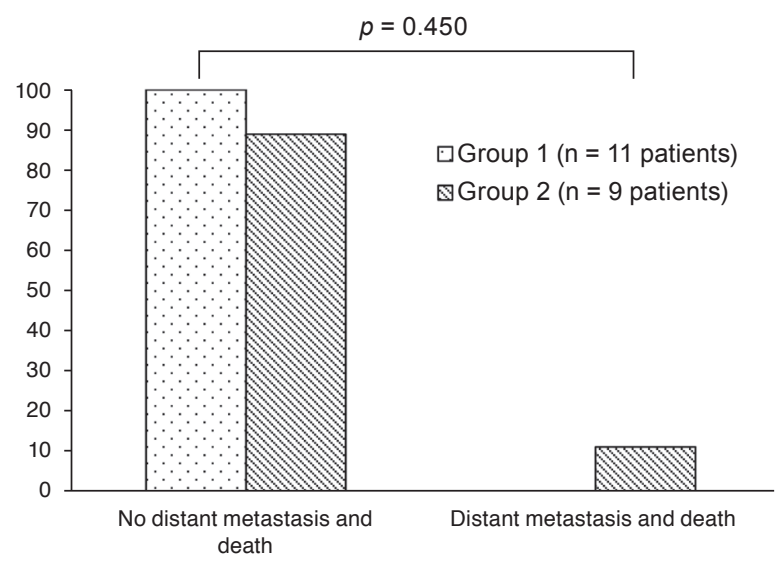

Fig. 1. Diagnoses and long-term treatment outcomes for retinoblastoma according to the age at diagnosis. (A) The eyes of patients diagnosed at age 1 year or older (group 2) had significantly higher International Intraocular Retinoblastoma Classification (IIRC) stages ( $p$ for trend $=0.010$, Cochran-Armitage test). (B,C) Patients diagnosed at age 1 year or older (group 2) had a significantly higher proportion of eyes with optic nerve invasion and eyes treated with enucleation ( $p=0.033$ and 0.046 , respectively, Fisher exact test). (D) The proportion of patients with distant metastasis and death were not significantly different according to the age at diagnosis $(p=0.450$, Fisher exact test). Group 1, age at diagnosis $<1$ year; group 2, age at diagnosis $\geq 1$ year.

using FISH had an initial manifesting dysmorphic feature sign. Patient numbers 19 and 20, who were diagnosed at the oldest ages, presented with an initial symptom of decreased visual acuity. Patient number 19 was initially diagnosed as having retinal detachment with proliferative vitreoretinopathy and was under simple observation without treatment for 19 months and was only diagnosed with retinoblastoma on the basis of histopathologic results after being enucleated because of increasing ocular pain.

The diagnoses and long-term treatment outcomes of non-familial retinoblastoma according to the age at diagnosis are shown in Fig. 1. The eyes of the patients in group 2 had a significantly higher IIRC stage than did those of the patients in group 1 (in group 1 with group C, 10.0\%; group $\mathrm{D}, 10.0 \%$; group $\mathrm{E}, 80.0 \%$ vs. group 2 with group $\mathrm{A}$,
$15.8 \%$; group $\mathrm{B}, 10.5 \%$; group $\mathrm{C}, 26.3 \%$; group $\mathrm{D}, 21.1 \%$; group E, 26.3\%; $p$ for trend = 0.010) (Fig. 1A). Moreover, the age at diagnosis (months) had a strong positive correlation with IIRC stage (Spearman's rho $=0.562, p=0.002$ ), whereas the lag time until diagnosis (months) in our study patients was significantly correlated with IIRC stage (Spearman's rho $=0.241, p=0.208$ ). The proportion of eyes with optic nerve invasion was also significantly higher in group 2 than in group $1(30.0 \%$ vs. $0.0 \%, p=0.033)$ (Fig. 1B). Compared to group 1, group 2 had a significantly higher proportion of eyes that were enucleated $(70.0 \%$ vs. $26.3 \%, p=0.046$ ) (Fig. 1C). In our study, none of the patients with bilateral retinoblastoma required bilateral enucleation. All patients, except for one patient with unilateral retinoblastoma without optic nerve involvement who had 
her eye enucleated, were treated with chemotherapy. The chemotherapy regimen included a combination of vincristine, carboplatin, and etoposide, with and without cyclophosphamide or ifosfamide. In all of the eyes with retinoblastoma that were not enucleated, local therapy using cryotherapy, laser photocoagulation, or both was administered along with systemic chemotherapy. External-beam radiation therapy was not performed in any of the patients, and none of them developed second tumors during follow-up. Although one patient diagnosed with group E retinoblastoma at 15 months, whose parents persistently refused enucleation, died because of obstructive hydrocephalus due to brain metastasis, no significant difference was observed in survival according to the age at diagnosis (88.9\% for group 2 vs. $100.0 \%$ for group $1, p=$ 0.450) (Fig. 1D).

Age at diagnosis (months) was significantly associated with germline $R B 1$ mutation (odds ratio [OR], $0.824 ; 95 \%$ confidence interval [CI], 0.689-0.986; $p=0.034$ ), but not with nerve invasion (OR, 6.226; 95\% CI, not estimated; $p$ $=0.549)$ or foveal involvement (OR, 1.087; 95\% CI, $0.943-$ $1.252 ; p=0.249$ ). Germline $R B 1$ mutation did not show significant correlations with IIRC stage (Spearman's rho = $-0.301, p=0.143$ ), nerve invasion (OR and 95\% CI were not estimated, $p=0.947$ ), or foveal involvement (OR, 0.375; 95\% CI, 0.063-2.244; $p=0.283$ ).

In this study, foveal involvement was found in 13 of the 19 eyes (68.4\%) in group 1 and 9 of the 10 eyes $(90.0 \%)$ in group 2, with no significant intergroup difference ( $p=$ 0.367). Of the 29 eyes of 20 patients in this study, 17 eyes of 13 patients were salvaged. After excluding five patients with mental retardation or who were too young to undergo visual acuity measurement, eight eyes of eight patients were considered suitable for measuring the BCVA at the final visit. The eyes with retinoblastoma but without foveal involvement had a significantly better visual acuity, with $75 \%$ of eyes having equivalent or better BCVA than Snellen $20 / 25$, than did those with foveal involvement, all of which had Snellen BCVA worse than $20 / 200(p=0.014)$.

\section{Discussion}

In this study, the clinical features and the clinical outcomes of non-screened retinoblastoma patients depending on the age at diagnosis were evaluated. We only included patients without a family history of retinoblastoma, and all were diagnosed because of a certain symptom or sign. Patients with retinoblastoma who were diagnosed at an age younger than 1 year were highly likely to have bilateral retinoblastoma with a germline $R B 1$ mutation (Table 1, 2). However, interestingly, they were diagnosed at a less advanced stage and classified as having a lower IIRC stage than were those diagnosed at an older age, thereby resulting in a better long-term prognosis in terms of less optic nerve invasion and less requirement for enucleation (Fig. 1), although the lag time until diagnosis did not differ between the two groups. This is unusual for a cancer that may develop due to a germline mutation, as germline mutations such as the BRCA1/2 mutation in breast cancer often suggest worse prognoses $[9,10]$. Survival did not differ according to the age at diagnosis (Fig. 1). The age at diagnosis (months) also showed a strong positive correlation with IIRC stage.

To our knowledge, the present analysis is the first to show that Korean retinoblastoma patients diagnosed at less than 1 year of age showed a tendency to be classified in a lower IIRC stage than did those diagnosed at an older age and that the age at diagnosis is significantly positively correlated with IIRC stage. In a similar context, a few recent studies reported in other parts of Asia showed that a higher proportion of patients with bilateral retinoblastoma rather than unilateral retinoblastoma were diagnosed at an earlier IIRC stage $[12,13]$. As in our study, these studies also showed that patients diagnosed at an age younger than 1 year were more likely to have bilateral retinoblastoma rather than unilateral retinoblastoma $[12,13]$.

The most common initial symptom or sign that led to presentation differed according to patient age at diagnosis in this study. Among the patients in group 1, strabismus was the most common condition (36.4\%), followed by leukocoria (27.3\%), and dysmorphic features (27.3\%), while among the patients in group 2, leukocoria was the most common condition (66.7\%). Many previous studies reported (with consensus) that the most common presenting symptom or sign of retinoblastoma was leukocoria $[7,12,14]$, which was in overall agreement with our study findings, but none of these studies had evaluated presenting signs and symptoms according to the age at diagnosis. In a study conducted in the USA, leukocoria was associated with more advanced disease, while strabismus was associated with macular involvement [14]. This association is 
consistent with our results, in that patients diagnosed at age 1 year or older presented with a much higher proportion of leukocoria and were classified in higher IIRC stages than were those diagnosed at a younger age. However, foveal involvement was also more frequently seen in those diagnosed at age 1 year or older in our study, even though most of them showed leukocoria rather than strabismus as their initial presenting sign or symptom. Dysmorphic features were the initial sign for those diagnosed at the youngest age and appeared as part of $13 q$ deletion syndrome, which also includes anteverted ear lobes, high and broad forehead, prominent philtrum, and severe mental retardation [15]. Large deletions in the $R B 1$ gene were detected using FISH in these patients. Decreased visual acuity was the initial symptom for two patients $(10 \%)$ diagnosed at the oldest ages, i.e., at 85 and 89 months. This is consistent with the findings of previous studies, which reported that poor vision comprises $7.7 \%$ of the presenting symptoms [14], and that low vision was the most common presenting sign in children diagnosed at an age older than 5 years [12].

In our study, germline $R B 1$ mutation was not significantly associated with disease severity in terms of IIRC stage and nerve invasion. Germline $R B 1$ mutations are detected in around $90 \%$ of patients with bilateral and/or familial retinoblastoma and $10 \%$ of patients with non-familial unilateral retinoblastoma $[16,17]$. In recent studies, a combination of PCR-direct sequencing of all 27 exons and their intronic flanking regions of the $R B 1$ gene along with MLPA to detect large deletions and duplications seems to be the conventional method $[16,18,19]$, with a high detection rate of around 95\% [19]. Although no single method is fully sensitive and efficient to detect $R B 1$ mutations, most germline $R B 1$ mutations are very small deletions, insertions, or point mutations [20], and therefore, direct sequencing is considered the single most useful method, with a detection rate around $70 \%$. Cytogenetic testing techniques such as FISH and MLPA are relatively simple to perform, but since they detect only large chromosomal deletions, only approximately $7 \%$ of $R B 1$ mutations can be detected $[21,22]$. This explains why we were unable to detect $R B 1$ mutations in three patients with bilateral retinoblastomatwo of them were tested only by using FISH. Additional testing with direct sequencing will likely reveal $R B 1$ mutations in these patients.

Enucleation is typically recommended for the eyes with retinoblastoma classified as IIRC group E, some eyes with advanced group D, and eyes with suspected extra-ocular extension [23]. Although enucleation cannot be performed sometimes because of rejection by the patient's family, it is often required in advanced cases, because late removal of IIRC group E eyes may increase mortality [24], as in one of our patients who died because of brain metastasis after persistent refusal of enucleation. In our study, among the patients in group 2, 30\% of the eyes had optic nerve invasion and $70 \%$ of the eyes were enucleated. In contrast, among the patients in group 1, none of the eyes had optic nerve invasion and only $26.3 \%$ of the eyes were enucleated, thus showing a significant difference from those diagnosed at an older age. Although none of the published studies examined the enucleation rate according to the age at diagnosis, some have examined it according to laterality. In agreement with our study, studies conducted in China and Malaysia revealed that approximately $80 \%$ to $90 \%$ of the eyes with unilateral retinoblastoma were enucleated; this is a significantly higher proportion compared to the $40 \%$ to $50 \%$ enucleation rate in the eyes with bilateral retinoblastoma $[12,13]$.

All patients in this study, except for one, were treated using chemotherapy. In all of the eyes that were not enucleated, consolidation therapy using laser photocoagulation and cryotherapy was performed. Chemotherapy along with focal treatment has been shown to be effective in previous studies, even in advanced cases [25]. This helps to avoid external-beam radiation therapy as in our study, as radiation therapy has been reported to increase the risk of a second cancer in the radiation field, especially in patients with germline $R B 1$ mutations [26], which may affect survival.

In our study, $75 \%$ of the eyes without foveal involvement had a final Snellen BCVA equivalent to or better than 20 / 25 , which is a visual acuity expected in normal eyes, even though all eyes with foveal involvement had a Snellen BCVA worse than $20 / 200$. This result is consistent with that of a previous study, which reported that $67 \%$ of eyes with extrafoveal tumors had a final BCVA equal to or better than a Snellen visual acuity of $6 / 12$ [27]. Therefore, patients with retinoblastoma who do not need enucleation can expect good vision after sufficient treatment if the tumor does not involve the fovea.

When interpreting our results, the following limitations should be considered. First, this study was a retrospective 
study, and we had to depend on the availability and accuracy of the medical records and examinations. In particular, the genetic analyses for most of the patients were insufficient and showed a low detection rate. A complete analysis with both direct sequencing of the $R B 1$ gene and cytogenetic testing would have yielded more useful results. Second, this was a single-institution study with a small sample size owing to the low prevalence of retinoblastoma. Third, there is a possibility that the differences found between groups 1 and 2 may be partially due to the differences in disease duration. However, in our study, there were no intergroup differences in the duration between the initiation of signs or symptoms until the diagnosis of retinoblastoma, and no significant correlation between the lag time and IIRC stage, which may be because the lag time was short in most patients. This suggests that delayed diagnosis is not likely the main reason for the difference in prognosis between the two groups. Despite these constraints, it is meaningful to report that the patients diagnosed at an early age with likely germline $R B 1$ mutations had a more favorable outcome than those with later onset retinoblastoma with likely somatic mutations. Since genetic testing for the $R B 1$ gene is not widely available, an estimation of prognosis according to the age at diagnosis may be helpful in some institutions. Also, the present study was the first to examine the clinical characteristics and longterm prognoses of Korean retinoblastoma patients according to their age at diagnosis.

In conclusion, patients diagnosed with retinoblastoma at an age of 1 year or older were likely to have somatic $R B 1$ mutations with unilateral retinoblastoma that was classified in a higher IIRC stage, had a higher proportion of optic nerve invasion, and had a higher requirement for enucleation than patients diagnosed at an age younger than 1 year, who were likely to have germline $R B 1$ mutations with bilateral retinoblastoma. Unlike some other cancers caused by germline genetic mutations, germline $R B 1 \mathrm{mu}-$ tation with early onset retinoblastoma does not seem to suggest a poor prognosis in Korean retinoblastoma patients.

\section{Conflict of Interest}

No potential conflict of interest relevant to this article was reported.

\section{References}

1. Park SJ, Woo SJ, Park KH. Incidence of retinoblastoma and survival rate of retinoblastoma patients in Korea using the Korean National Cancer Registry database (1993-2010). Invest Ophthalmol Vis Sci 2014;55:2816-21.

2. Broaddus E, Topham A, Singh AD. Incidence of retinoblastoma in the USA: 1975-2004. Br J Ophthalmol 2009;93:21-3.

3. Kivela $T$. The epidemiological challenge of the most frequent eye cancer: retinoblastoma, an issue of birth and death. Br J Ophthalmol 2009;93:1129-31.

4. Corson TW, Gallie BL. One hit, two hits, three hits, more?: genomic changes in the development of retinoblastoma. Genes Chromosomes Cancer 2007;46:617-34.

5. Soliman SE, Dimaras H, Khetan V, et al. Prenatal versus postnatal screening for familial retinoblastoma. Ophthalmology 2016;123:2610-7.

6. Chantada GL, Qaddoumi I, Canturk S, et al. Strategies to manage retinoblastoma in developing countries. Pediatr Blood Cancer 2011;56:341-8.

7. Asencio-Lopez L, Torres-Ojeda AA, Isaac-Otero G, Leal-Leal CA. Treating retinoblastoma in the first year of life in a national tertiary paediatric hospital in Mexico. Acta Paediatr 2015;104:e384-7.

8. Draper GJ, Sanders BM, Brownbill PA, Hawkins MM. Patterns of risk of hereditary retinoblastoma and applications to genetic counselling. Br J Cancer 1992;66:211-9.

9. Baretta Z, Mocellin S, Goldin E, et al. Effect of BRCA germline mutations on breast cancer prognosis: a systematic review and meta-analysis. Medicine (Baltimore) 2016;95:e4975.

10. Wang YA, Jian JW, Hung CF, et al. Germline breast cancer susceptibility gene mutations and breast cancer outcomes. BMC Cancer 2018;18:315.

11. Linn Murphree A. Intraocular retinoblastoma: the case for a new group classification. Ophthalmol Clin North Am 2005;18:41-53.

12. Gao J, Zeng J, Guo B, et al. Clinical presentation and treatment outcome of retinoblastoma in children of South Western China. Medicine (Baltimore) 2016;95:e5204.

13. Subramaniam S, Rahmat J, Rahman NA, et al. Presentation of retinoblastoma patients in Malaysia. Asian Pac $J$ Cancer Prev 2014;15:7863-7.

14. Abramson DH, Frank CM, Susman M, et al. Presenting signs of retinoblastoma. J Pediatr 1998;132(3 Pt 1):505-8.

15. Baud O, Cormier-Daire V, Lyonnet S, et al. Dysmorphic phenotype and neurological impairment in 22 retinoblasto- 
ma patients with constitutional cytogenetic $13 \mathrm{q}$ deletion. Clin Genet 1999;55:478-82.

16. Dommering CJ, Mol BM, Moll AC, et al. RB1 mutation spectrum in a comprehensive nationwide cohort of retinoblastoma patients. J Med Genet 2014;51:366-74.

17. Price EA, Price K, Kolkiewicz K, et al. Spectrum of RB1 mutations identified in 403 retinoblastoma patients. $J$ Med Genet 2014;51:208-14.

18. Nguyen HH, Nguyen HT, Vu NP, et al. Mutational screening of germline RB1 gene in Vietnamese patients with retinoblastoma reveals three novel mutations. Mol Vis 2018;24:231-8.

19. Seo SH, Ahn HS, Yu YS, et al. Mutation spectrum of RB1 gene in Korean bilateral retinoblastoma patients using direct sequencing and gene dosage analysis. Clin Genet 2013;83:494-6.

20. Harbour JW. Overview of RB gene mutations in patients with retinoblastoma: implications for clinical genetic screening. Ophthalmology 1998;105:1442-7.

21. Richter S, Vandezande K, Chen N, et al. Sensitive and efficient detection of RB1 gene mutations enhances care for families with retinoblastoma. Am J Hum Genet 2003;72: 253-69.
22. Bunin GR, Emanuel BS, Meadows AT, et al. Frequency of 13q abnormalities among 203 patients with retinoblastoma. J Natl Cancer Inst 1989;81:370-4.

23. Ghassemi F, Khodabande A. Risk definition and management strategies in retinoblastoma: current perspectives. Clin Ophthalmol 2015;9:985-94.

24. Zhao J, Dimaras H, Massey C, et al. Pre-enucleation chemotherapy for eyes severely affected by retinoblastoma masks risk of tumor extension and increases death from metastasis. J Clin Oncol 2011;29:845-51.

25. Shields CL, Shields JA, Needle M, et al. Combined chemoreduction and adjuvant treatment for intraocular retinoblastoma. Ophthalmology 1997;104:2101-11.

26. Kleinerman RA, Tucker MA, Tarone RE, et al. Risk of new cancers after radiotherapy in long-term survivors of retinoblastoma: an extended follow-up. J Clin Oncol 2005;23:2272-9.

27. Narang S, Mashayekhi A, Rudich D, Shields CL. Predictors of long-term visual outcome after chemoreduction for management of intraocular retinoblastoma. Clin Exp Ophthalmol 2012;40:736-42. 